\title{
HelpCare: Um Protótipo de ChatBot para o Auxílio do Tratamento de Doenças Crônicas
}

\author{
Natália Oliveira $^{1}$, Allan Costa ${ }^{1}$, Diovanni Araújo ${ }^{1}$, Carlos Portela $^{1}$ \\ ${ }^{1}$ Faculdade de Sistemas de Informação (FASI) - Universidade Federal do Pará (UFPA) \\ Cametá - PA - Brasil \\ nataliatatieleufpa@gmail.com, \{allancosta, diovanni,csp\}@ufpa.br
}

\begin{abstract}
The effective involvement of patients in treatment is a big challenge for all health professionals, especially when dealing with chronic diseases. The treatment of these diseases involves lifestyle changes and a continuous care process. In this context, the use of chatbots allows to offer more personalized care for each patient, reducing the waiting time for basic care and the lack of constant monitoring. This paper proposes a chatbot prototype, called HelpCare, developed through the IBM Watson cognitive computing tool. This prototype aims to help patients with chronic diseases, such as diabetes, high cholesterol and hypertension.
\end{abstract}

Resumo. O envolvimento efetivo dos pacientes no tratamento é um grande desafio para todas os profissionais de saúde, principalmente quando se trata de doenças crônicas. O tratamento dessas doenças envolve mudanças de estilo de vida e um processo de cuidado contínuo. Neste contexto, o uso de chatbots permite oferecer cuidados mais personalizados para cada paciente, reduzindo o tempo de espera por atendimento básico e a falta de acompanhamento constante. Este artigo apresenta um protótipo de chatbot, denominado HelpCare, desenvolvido através da ferramenta de computação cognitiva IBM Watson. Esse protótipo é direcionado a auxiliar pacientes com doenças crônicas, como diabetes, colesterol alto e hipertensão arterial.

\section{Introdução}

De acordo com um estudo realizado por pesquisadores da Faculdade de Medicina da Universidade de São Paulo, um terço dos casos de demência diagnosticados no Brasil poderia ser evitado com o controle de doenças crônicas como hipertensão e obesidade [Estadão 2017]. As doenças crônicas, segundo a Portaria no 483 do Ministério da Saúde (2014), são aquelas que apresentam início gradual, com duração longa ou incerta, que, em geral, apresentam múltiplas causas e cujo tratamento envolve mudanças de estilo de vida e um processo de cuidado contínuo que, usualmente, não leva à cura.

Neste contexto, observa-se que o envolvimento efetivo dos pacientes é um grande desafio para todos os profissionais de saúde, tendo em vista as possíveis consequências geradas, devido à falta de comprometimento com o tratamento. Esse envolvimento pode ser obtido através do apoio de um sistema computacional que permita captar, transmitir, receber, armazenar, distribuir, interligar e manipular dados para os principais objetivos de um serviço de atenção à saúde [Murphy, Hanken e Waters 1999]. 
Sendo assim, esta pesquisa propõe um protótipo de chatbot, denominado HelpCare, desenvolvido a partir da ferramenta de computação cognitiva IBM Watson, a fim de auxiliar pacientes com doenças crônicas como diabetes, colesterol alto e hipertensão arterial. O termo "chatbot" designa uma tendência nos assistentes pessoais inteligentes acionados por comandos, como a troca de mensagens por texto, capazes de entender seres humanos [Boldo 2016]. Estas tecnologias vêm permeando nas esferas da Tecnologia da Informação (TI) e da área de Saúde, caracterizando-se como sistemas cognitivos que, segundo Cervenka et al. (2016), buscam simular funções do cérebro humano como: tomada de decisão, processamento de informação e comunicação oral.

Além dessa seção introdutória, a Seção 2 apresenta a fundamentação teórica que descreve os principais conceitos da computação cognitiva e o uso de chatbots, com destaque para o IBM Watson. Na Seção 3 são apresentadas as funcionalidades do HelpCare e os seus protótipos de tela. Por fim, a Seção 4 apresenta os resultados parciais, bem como as etapas futuras a serem realizadas.

\section{Computação Cognitiva}

A computação cognitiva define uma coleção de tecnologias emergentes inspiradas no processamento biológico da informação pelo sistema nervoso, no raciocínio humano, na tomada de decisões e na seleção natural [Brasil, Azevedo e Barreto 2001]. É composta por técnicas de Inteligência Artificial (IA), como processamento de linguagem natural, geração de hipóteses baseada em evidências, aprendizado de máquina e dezenas de outros algoritmos e tecnologias que analisam as evidências em diferentes dimensões.

Para Feldman e Reynolds (2014), os sistemas cognitivos devem apresentar:

- Aprendizagem dinâmica e adaptativa a partir de mudanças permanentes de informação;

- Análise probabilística por buscarem a descoberta de novos padrões baseados no contexto e na criação de hipóteses baseadas em evidências;

- Processamento de dados baseado em significado com o uso de linguagens natural e semântica;

- Interatividade por contemplarem ferramentas para comunicação e interação com humanos, bem como para análise e visualização de dados.

\subsection{ChatBots}

$\mathrm{Na}$ área de Saúde, os chatbots podem desempenhar um papel estratégico na personalização do atendimento ao usuário e na melhoria da retenção e envolvimento dos pacientes. Os "bots" podem estar disponíveis 24 horas por dia para oferecer acesso ao paciente a todos os tipos de suporte de saúde, como por exemplo o agendamento de medicação [Iyer 2017]. Esse suporte personalizado pode reduzir o tempo de espera e a falta de acompanhamento dos pacientes.

Um chatbot é composto de linhas de código, e pode residir em qualquer um dos populares aplicativos de mensagens sociais usados atualmente. O usuário só precisa adicionar o bot como seu contato no aplicativo de mensagens de sua escolha e começar a conversar. Assim, o aprendizado é gerado a partir de cada conversa realizada e o resultado é uma interação cada vez melhor com as pessoas em conversas futuras. 
Diante disto, existem alguns estudos realizados no contexto de ChatBot, bem como aplicações já desenvolvidas, porém em grande maioria em outros idiomas e para outros tipos de serviços. O Serviço Nacional de Saúde do Reino Unido, por exemplo, usa um aplicativo de chatbot para dispensar conselhos médicos com o objetivo de reduzir o fardo em suas 111 linhas de ajuda não emergenciais. Assim, desenvolveu o aplicativo Babylon Health, um serviço médico sob demanda [Medicalfuturist 2017].

\subsection{IBM Watson}

O IBM Watson [IBM 2019], criado em meados de 2006, foi uma inovação para os sistemas cognitivos. O que caracteriza o Watson é a combinação de três capacidades:

- Processamento de linguagem natural, ajudando a entender as complexidades dos dados não estruturados, que representam até $80 \%$ dos dados no mundo atual;

- Geração e avaliação de hipóteses pela aplicação de análises avançadas para pesar e avaliar um painel de respostas baseado apenas na evidência relevante;

- Aprendizagem baseada na evidência, que se aprimora a partir dos resultados e se tornar cada vez mais inteligente com cada repetição e interação.

Assim, os mecanismos de IA que serão utilizados no HelpCare são: aprendizagem de máquina, que usa os dados para detectar padrões e ajustar as ações da máquina; e análise dos dados, estruturados ou não-estruturados, que são coletados, armazenados e interpretados por sistemas especializados.

A capacidade do Watson está no entendimento do significado e do contexto da linguagem humana para processar rapidamente informações e encontrar respostas precisas para perguntas complexas. Isto reflete o seu grande potencial para transformar a forma como os computadores podem ajudar as pessoas na área de Saúde. Adicionalmente, trata-se de uma solução open source que permite um diálogo mais humano e pode usar o aprendizado automático interativo e supervisionado.

\section{HelpCare}

\subsection{Requisitos}

O Quadro 1 apresenta os Requisitos Funcionais (RFs) do aplicativo HelpCare. De acordo com Pressman (2016), os RFs são as funções que o sistema deve fornecer, ou seja, como deverá reagir as entradas do usuário.

Quadro 1. Requisitos Funcionais do HelpCare

\begin{tabular}{|l|l|l|}
\hline ID & Nome & Descrição \\
\hline RF01 & Tirar dúvidas & $\begin{array}{l}\text { O aplicativo deve permitir tirar dúvidas sobre a doença } \\
\text { crônica que o usuário possui. }\end{array}$ \\
\hline RF02 & $\begin{array}{l}\text { Avisar horários das } \\
\text { medicações }\end{array}$ & $\begin{array}{l}\text { O aplicativo deve auxiliar no gerenciamento dos horários de } \\
\text { remédios, onde o paciente poderá registrar os mesmos. }\end{array}$ \\
\hline RF03 & $\begin{array}{l}\text { Cadastrar finalidade } \\
\text { dos remédios }\end{array}$ & $\begin{array}{l}\text { O aplicativo deve permitir ao usuário cadastrar o propósito } \\
\text { dos remédios, relacionando-os com suas doenças crônicas. }\end{array}$ \\
\hline
\end{tabular}




\begin{tabular}{|l|l|l|}
\hline RF04 & Auxiliar na dieta & $\begin{array}{l}\text { O aplicativo deve auxiliar na dieta do usuário, através de } \\
\text { dicas e avisos, de acordo com suas doenças crônicas. }\end{array}$ \\
\hline RF05 & $\begin{array}{l}\text { Fornecer feedback } \\
\text { sobre resultados }\end{array}$ & $\begin{array}{l}\text { O aplicativo deve prestar assistência com os resultados da } \\
\text { verificação de pressão arterial e glicemia. }\end{array}$ \\
\hline RF06 & Armazenar histórico & $\begin{array}{l}\text { O aplicativo deve armazenar o histórico de assistências } \\
\text { realizadas para o usuário. }\end{array}$ \\
\hline RF07 & Listar hospitais & $\begin{array}{l}\text { O aplicativo deve mostrar os hospitais mais próximos e } \\
\text { números de emergência médica da região. }\end{array}$ \\
\hline
\end{tabular}

\subsection{Protótipos de Tela}

Após definidos os requisitos, foram construídos os protótipos de tela de alta fidelidade. Rocha et al. (2018) destacam que o Departamento de Saúde e Serviços Humanos dos Estados Unidos utiliza este tipo de protótipo a fim de agregar uma experiência de interação o mais real possível com a interface da aplicação. Assim, foi escolhido como ferramenta IBM Watson Assistant [IBM 2019].

A emulação do chatbot realizou-se por meio da área de experimentação do próprio IBM Watson Assistant. As telas do protótipo exibidas na Figura 1 demostram a usabilidade da ferramenta de forma simples, onde o usuário inicia uma conversação com o chatbot, por meio de uma saudação (a), logo após irá selecionar qual sua doença (b), o tipo específico (c). Por meio dessa interação, o bot informará mais sobre a doença, desde os sintomas (d) e possíveis tratamentos (e).

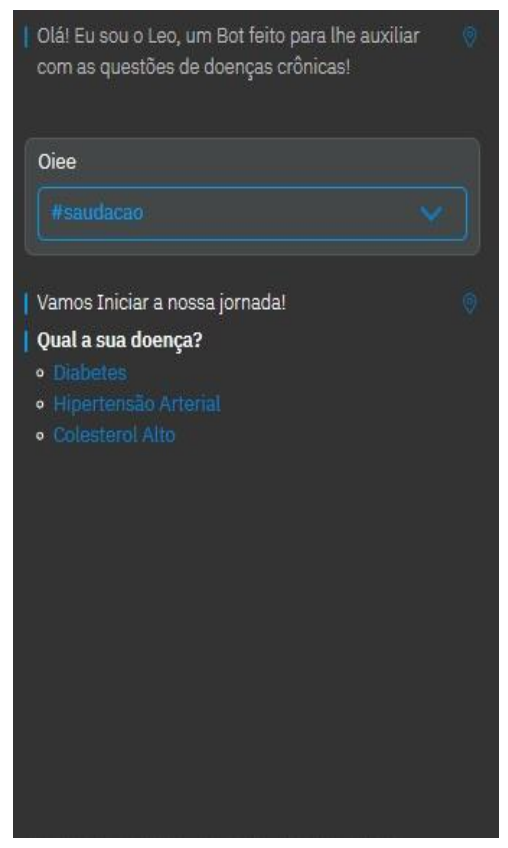

A) Início do chat

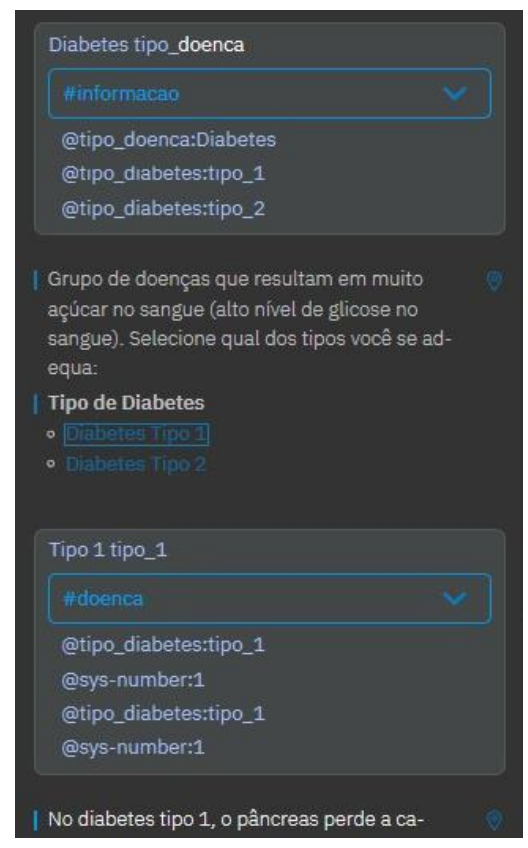

B) Seleção da doença

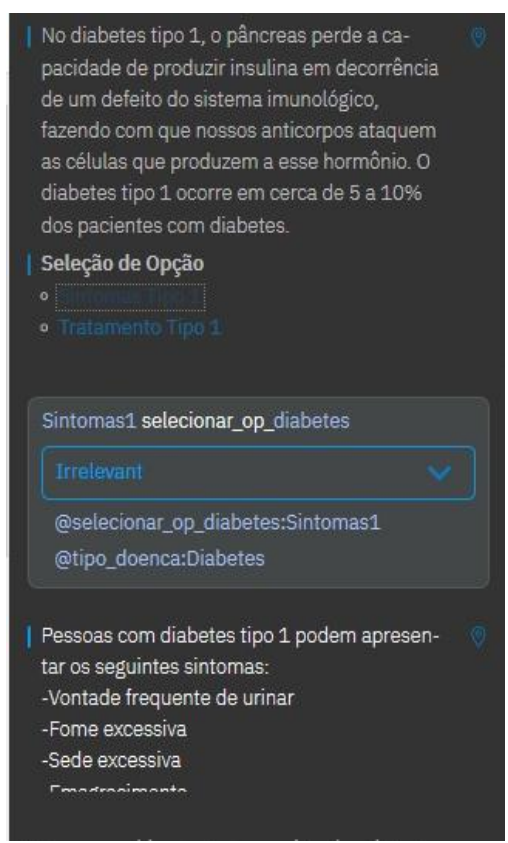

C) Seleção do tipo de diabetes 


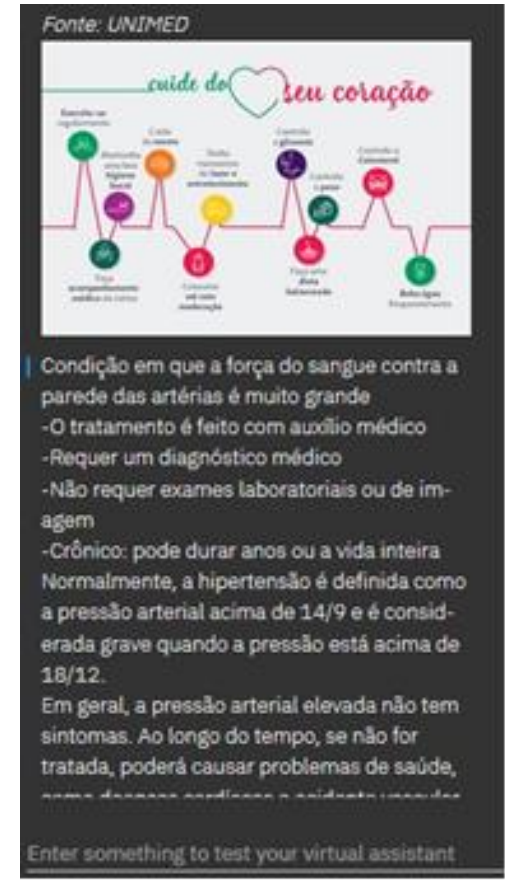

D) Informações sobre a doença

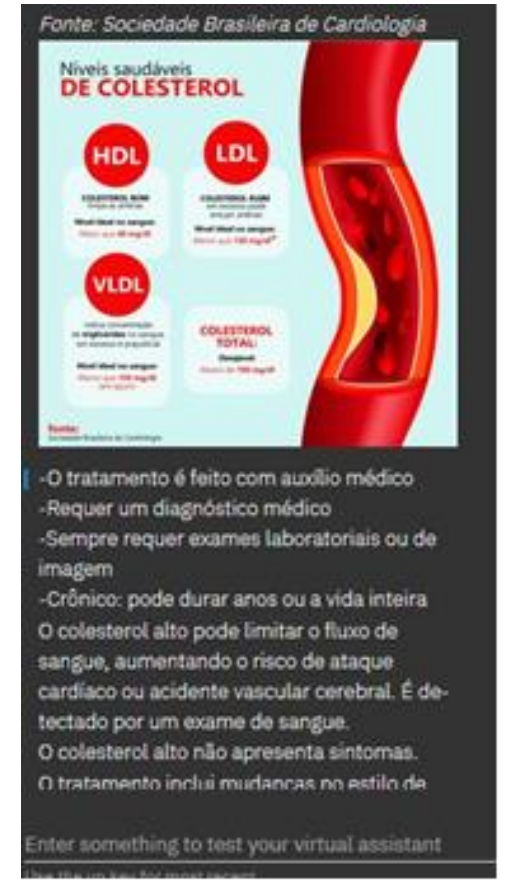

E) Informações sobre o tratamento

A computação cognitiva realizada no protótipo consegue identificar a classificação do que foi inserido pelo usuário, tanto texto quanto a escolha de opções. Como por exemplo uma saudação - "Oi”, como também a partir do momento que o usuário inserir uma saudação diferente o IBM Watson, inicia o seu aprendizado determinando essa nova palavra como uma saudação. As doenças também são classificadas em suas classes, mostrando assim a funcionalidade correta da ferramenta.

\subsection{Apoio ao Tratamento de Diabetes}

Um paciente diabético precisa medir seus níveis de glicose no sangue diariamente. Neste sentido, um sistema de IA pode armazenar as informações de cada medição, analisar e gerar insights para o cuidado futuro do paciente [TNH Health 2019]. O HelpCare buscará prever a ocorrência de uma hipoglicemia antes dela acontecer, dando ao usuário a possibilidade de se preparar para o tratamento. Além disso, o aplicativo poderá ser abastecido com informações sobre a doença, de modo a atuar como um assistente para os pacientes. Desta forma, poderá tirar dúvidas sobre suas condições, propor ações remediativas e responder outras perguntas relacionada à diabetes.

\section{Considerações Finais}

O objetivo deste trabalho foi apresentar um projeto para o desenvolvimento de uma aplicação para o tratamento de doenças crônicas, considerando as principais dificuldades das pessoas que sofrem destas doenças, juntamente com a tecnologia da computação cognitiva que é utilizada pelo chatbots. A ferramenta IBM Watson foi empregada para desenvolver um protótipo base para evidenciar a prática e facilidade de manuseio dos bots por parte dos chats juntamente com o usuário, uma vez que a sua interface é bastante similar com as conversações convencionais que o paciente realiza no seu dia a dia, como o uso de aplicativos de mensagens já contidos em seu aparelho de celular. 
Portanto, nesta pesquisa, observou-se que o conceito de chatbot é utilizado em diversas áreas para facilitar o atendimento aos usuários. Neste contexto, chatbots na área de Saúde podem auxiliar no controle e tratamento de doenças crônicas. Seus mecanismos e funcionalidades permitirão a oferta de aplicações, atuais e futuras, com maior grau de integração e interatividade entre os usuários e os serviços.

Considerando os resultados preliminares deste artigo, será desenvolvido, como trabalho futuro, uma aplicação de chatbot integrada ao mensageiro Telegram, tendo em vista o crescimento na utilização no mercado de aplicativos de mensagens. Algumas das vantagens do Telegram são, além dos seus 200 milhões de usuários ativos mensais, seus recursos avançados de segurança e criptografia [Demartini 2018].

\section{Referências}

Boldo, F. (2016) "3 Usos de ChatBot na Saúde”, http://saudebusiness.com/3-usos-doschatbots-em-saude, Janeiro.

Brasil, L., Azevedo, F. and Barreto, J. (2001). Hybrid expert system for decision supporting in the medical area: complexity and cognitive computing". In International Journal of Medical Informatics, v. 63, n. 1, pages 19-30.

Cervenka, P., Hlavaty, I., Miklosik, A. and Lipianska, J. (2016). Using cognitive systems in marketing analysis. In J. Economic Annals, v. 160, n. 7-8, pages 56- 61.

Dermatini, F. (2018) "Telegram chega a 200 milhões de usuários mensais", https://canaltech.com.br/apps/telegram-chega-a-200-milhoes-de-usuarios-mensais110532, Março.

Estadão (2017) “Controle de Doenças Crônicas pode evitar 1 em cada 3 casos de demência no Brasil”, https://noticias.uol.com.br/saude, Abril.

Feldman, S. and Reynolds, H. (2014). Cognitive computing: A definition and some thoughts. In KMWorld,v. 23, n. 10, November/December.

IBM (2019) “IBM Watson”, https://www.ibm.com/Watson/APIs, Janeiro.

Iyer, S. (2017) "Chatbots -The Perfect Way to Deliver Personalized Patient Care", https://chatbotsmagazine.com/chatbots-the-perfect-way-to-deliver-personalizedpatient-care-d1278287602d, Março.

Medicalfuturist (2017) "Chatbots Will Serve As Health Assistants", http://medicalfuturist.com/chatbots-health-assistants, April.

Ministério da Saúde (2014) "Portaria $\mathrm{n}^{\circ}$ 483, de $1^{\circ}$ de abril de 2014", http://bvsms.saude.gov.br/bvs/saudelegis/gm/2014/prt0483_01_04_2014.html, Abril.

Murphy, G., Hanken, M. A. and Waters, K. (1999), Electronic health records: Changing the vision, Saunders WB Co., $1^{\text {st }}$ edition.

Pressman, R. (2016), Engenharia de Software: Uma Abordagem Profissional, McGrawHill, Bookman, $8^{\text {a }}$ edição.

Rocha, M., Soares, R., Veras, M. e Santos W. (2018) "UnaSUS Conecta: Redes Sociais à Serviço da Saúde", In: Congresso Brasileiro de Informática em Saúde (CBIS), Fortaleza-CE.

TNH Health (2019) “I.A. e diabetes o que eles têm em comum?", http://tnh.health/blog/inteligencia-artificial-e-diabetes-2/, Abril. 